\title{
Multiple interactions at HERA
}

\author{
A. Knutsson ${ }^{\mathrm{a}}$ (on behalf of the H1 and ZEUS collaborations) \\ aDESY - Deutsches Elektronensynchrotron, Notkestr. 85, \\ 22607 Hamburg, Germany
}

Presented are recent measurements in $e p$ collisions at HERA, which comprise results that are expected to be sensitive to multiple interactions and the underlying event. A measurement of 3- and 4-jet event crosssections in photoproduction, as well as two measurements which target the activity in the soft underlying event in photoproduction and deep inelastic scattering at low photon virtuality $\left(Q^{2}\right)$, respectively, are reviewed. The data are compared to various QCD models with and without multiple interactions simulated. The multijet event cross-sections are in addition compared to a fixed-order $\mathcal{O}\left(\alpha \alpha_{s}^{2}\right)$ calculation.

\section{Introduction}

Neutral current electron-proton collisions at HERA can be divided into interactions with direct and resolved photons. In direct photon interactions the photon interacts as a point-like particle, while in the resolved photon interaction the photon fluctuates into a partonic system. The latter results in a reaction that resembles hadronhadron collisions, where interactions between the beam remnants may occur in addition to the primary ones. This is called multiple interactions (MI).

In photoproduction, where the exchanged photon theoretically is real and experimentally the photon virtuality, $Q^{2}$, is close to 0 , the partonic fluctuations of the photon can be long lived and the contribution to the cross section from resolved photons larger. The life time of the partonic fluctuation of the photon typically scales with $1 / Q$. Thus, in photoproduction and in low $Q^{2}$ deep inelastic scattering (DIS), additional remnant-remnant interactions can be expected to contribute to jet multiplicities and the underlying event (UE). The UE is defined as everything except the studied LO process, which is the hardest interaction in the event. Already more than 10 years ago, H1 and ZEUS have shown [1-3] that in photoproduction jet cross-sections and the UE can only be described by theoretical models, if MI are taken into consideration.
The fractional momentum of the exchanged photon carried by the parton taking part in the hard interaction, $x_{\gamma}^{\text {obs }}$, gives an experimental handle to select samples enriched in direct or resolved photon events. $x_{\gamma}^{\text {obs }}$ close to one means that predominantly a direct photon takes part in the reaction.

The situation is summarised in Fig. 1, where the number of dijet events in photoproduction is measured [3] as a function of $x_{\gamma}^{\text {obs }}$. The uncorrected data are compared to predictions from the Monte Carlo generators PYTHIA [4] and HERWIG [5] with MI, as well as HERWIG without MI. Also the direct photon contribution is shown separately for HERWIG. The Monte Carlo (MC) simulated events have gone through a full detector simulation. One can clearly see that only models with MI simulated are able to account for the data in the resolved enriched region.

Using a total integrated luminosity of $121 \mathrm{pb}^{-1}$ ZEUS measures 3- and 4-jet cross-sections in photoproduction [6]. This measurement provides a good tool to study higher-order QCD, such as fixed-order calculations and QCD models with parton showers, as well as the contributions from MI, which are expected to increase the multijet cross-section.

Two measurements from the $\mathrm{H} 1$ collaboration $[7,8]$ investigate the underlying event by measuring the activity in different azimuthal regions with respect to the hardest jet in the event, re- 


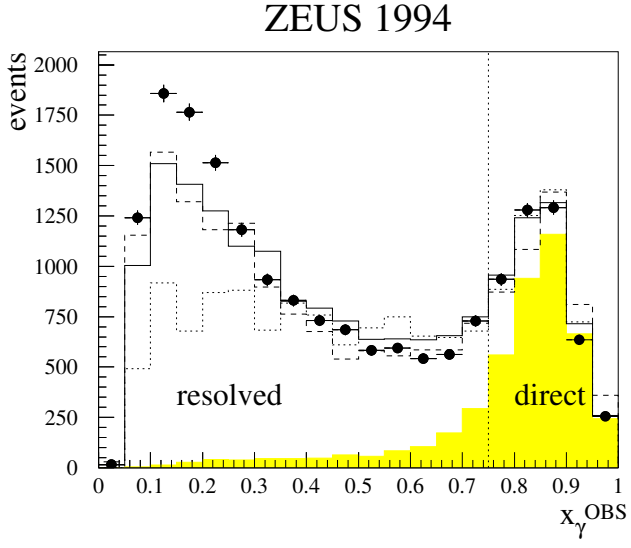

Figure 1. The number of uncorrected dijet events in photoproduction data [3] as a function $x_{\gamma}^{\text {obs }}$ compared to Monte Carlo predictions. The lines represent: PYTHIA with MI (dashed line), HERWIG with MI (full line), HERWIG without MI (dotted line). The contribution from direct photon processes in HERWIG is shown as a shaded area.

ferred to as the leading jet. Similar measurements have been performed at the TEVATRON [9]. In the first measurement, the charged particle multiplicity in dijet events is measured in photoproduction. In the second one, the production of jets with low transverse momenta, so called minijets, is measured in low $Q^{2}$ deep inelastic scattering events with at least one hard jet. Although the possibility for remnant-remnant interactions is smaller in DIS, where the contribution from resolved photons is smaller, one can expect secondary interactions between the proton remnant and the quarks produced at the photon vertex. The measurement is performed in bins of $Q^{2}$. This is useful in order to control the contribution from resolved photon events which is expected to decrease with increasing $Q^{2}$.

\section{Theoretical Models}

\subsection{Monte Carlo Programs}

The different measurements are compared to various theoretical predictions. The Monte Carlo programs RAPGAP 3.1 [10], HERWIG 6.5 [5] and PYTHIA 6.2 [4] are based on leading-order matrix elements supplemented with parton showers in the DGLAP scheme, while the ARIADNE [11] program is producing gluon cascades according to the Color Dipole Model (CDM) [11].

The PYTHIA 6.2 and HERWIG 6.5 programs allow to add contributions from remnant-remnant multiple interactions. In PYTHIA 6.2 this is done by using the so called simple model. In this model the total partonic interaction crosssection $\sigma_{i n t}\left(p_{t, \min }\right)$, integrated from a cut off $p_{t, \text { min }}$, grows with decreasing $p_{t, \text { min }}$ such that it finally exceeds the total non-diffractive crosssection $\sigma_{T o t}$. This is interpreted that the average number of partonic interactions $\langle n\rangle$ is larger than one, i.e. $\langle n\rangle=\sigma_{\text {int }}\left(p_{t, \min }\right) / \sigma_{\text {Tot }}[12]$.

In HERWIG 6.5, two different models for $\mathrm{UE} / \mathrm{MI}$ are available. The first is based on a phenomenological model for the soft underlying event as introduced by the UA5 collaboration; its main effect is to distribute additional energy isotropically in the rest frame of the UE. In additional, the JIMMY package can be used to simulate MI within the HERWIG framework. JIMMY 4.0 [13] implements an impact parameter dependent MI model, in which the colliding particles are approximated as overlapping discs. In the HERWIG predictions presented here, MI is simulated by using JIMMY 4.0.

These MI models are so far only implemented for remnant-remnant interactions, and although one can imagine additional scatterings between the proton remnant and the partons produced in a direct photon interaction, where no photon remnant is present, there are so far no attempts made to describe such MI events.

If not stated otherwise, the default parameters of the generators are used in the presented studies.

\section{2. $\mathcal{O}\left(\alpha \alpha_{s}^{2}\right)$ Calculation}

The 3-jet cross-sections measured by ZEUS in photoproduction are compared to a next-toleading order $\mathcal{O}\left(\alpha, \alpha_{s}^{2}\right)$ calculation $[14,15]$, which here effectively is a leading order calculation, since events with three jets are selected. The calculations are corrected for hadronisation and mul- 
tiple interaction effects using PYTHIA 6.2 and HERWIG 6.5.

\section{3- and 4-jet Final States in Photopro- duction}

The ZEUS collaboration has measured [6] 3and 4-jet event cross-sections in photoproduction. Analysed were events with the photon virtuality $Q^{2}<1 \mathrm{GeV}^{2}$ and the photon inelasticity $0.2<y<0.85$. Jets were defined according to the inclusive $k_{t}$-algorithm and were required to have transverse energy $E_{T}>6 \mathrm{GeV}$ and pseudorapidity $\left|\eta_{\text {jet }}^{\text {lab }}\right|<2.4$. The events were divided into two subsamples with respect to the invariant mass $M_{n j}$ of the $n$-jet system, $25<M_{n j}<50 \mathrm{GeV}$ and $M_{n j}>50 \mathrm{GeV}$, respectively.

In Fig. 2a) the 3-jet event cross-section is shown as a function of the invariant mass of the 3 -jet system, $M_{3 j}$. The statistical errors of the data are represented by the inner error bars, while the outer error bars represent the systematic and the statistical errors added in quadrature. The shaded band following the data points gives the systematic error from the calorimeter energy scale uncertainty. In this figure the cross-section is compared to the $\mathcal{O}\left(\alpha \alpha_{s}^{2}\right)$ calculation [14,15] (described in 2.2). The theoretical uncertainty on the calculation is indicated as the dashed band. Fig. 2b) shows the hadronisation and MI corrections. While the hadronisation corrections are small and constant, the MI corrections are increasing with lower invariant mass by up to a factor of 2. The symmetric uncertainties of the corrections are calculated as half of the difference between the predictions from HERWIG and PYTHIA. As can be seen, the uncertainty of the MI corrections are significantly larger than the hadronisation corrections, and decreases from $20 \%$ at low $M_{3 j}$ to about $5 \%$ at high $M_{3 j}$. In Fig. 2c), the ratio of data over theory is shown. The data are described within the fairly large theoretical uncertainty, but only when the calculation is corrected for MI effects.

The same cross-sections, and additionally the 4 -jet cross-sections, are shown in Fig. 3 as a function of the invariant mass of the 4-jet system, $M_{4 j}$, and are compared to HERWIG and

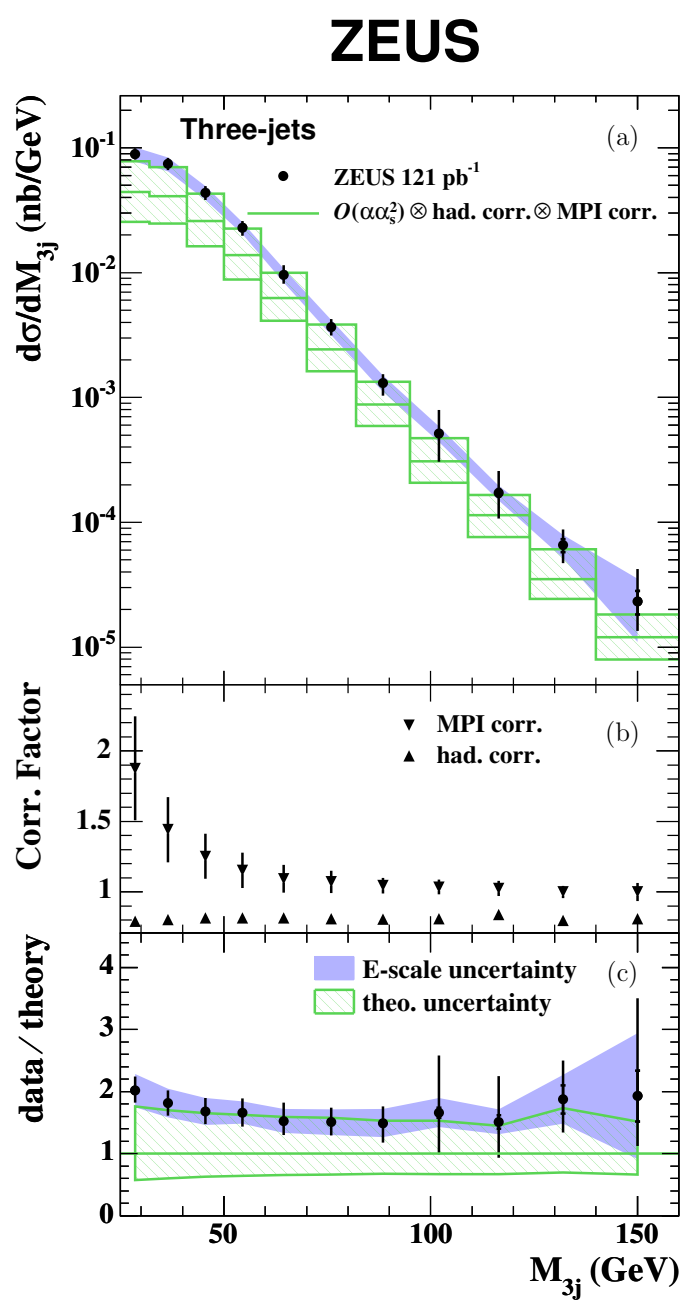

Figure 2. 3-jet cross-sections as a function of the invariant mass, $M_{3 j}$, of the 3 -jet system. The data are compared to a fixed-order $\mathcal{O}\left(\alpha \alpha_{s}^{2}\right)$ calculation. The middle plot shows the corrections applied to the calculation, and the lower plot shows the ratio between data and theory. [6]

PYTHIA with and without contributions from MI. Furthermore for HERWIG the direct photon contribution are showed separately. Each model prediction has been area normalised to the data in the high mass region $\left(M_{n j}>50 \mathrm{GeV}\right)$, as indicated by the scaling factors given in the legend. The result is a reasonable description of the shape 
of the data in the high mass region. However, at low invariant masses the data cross-sections are

ZEUS
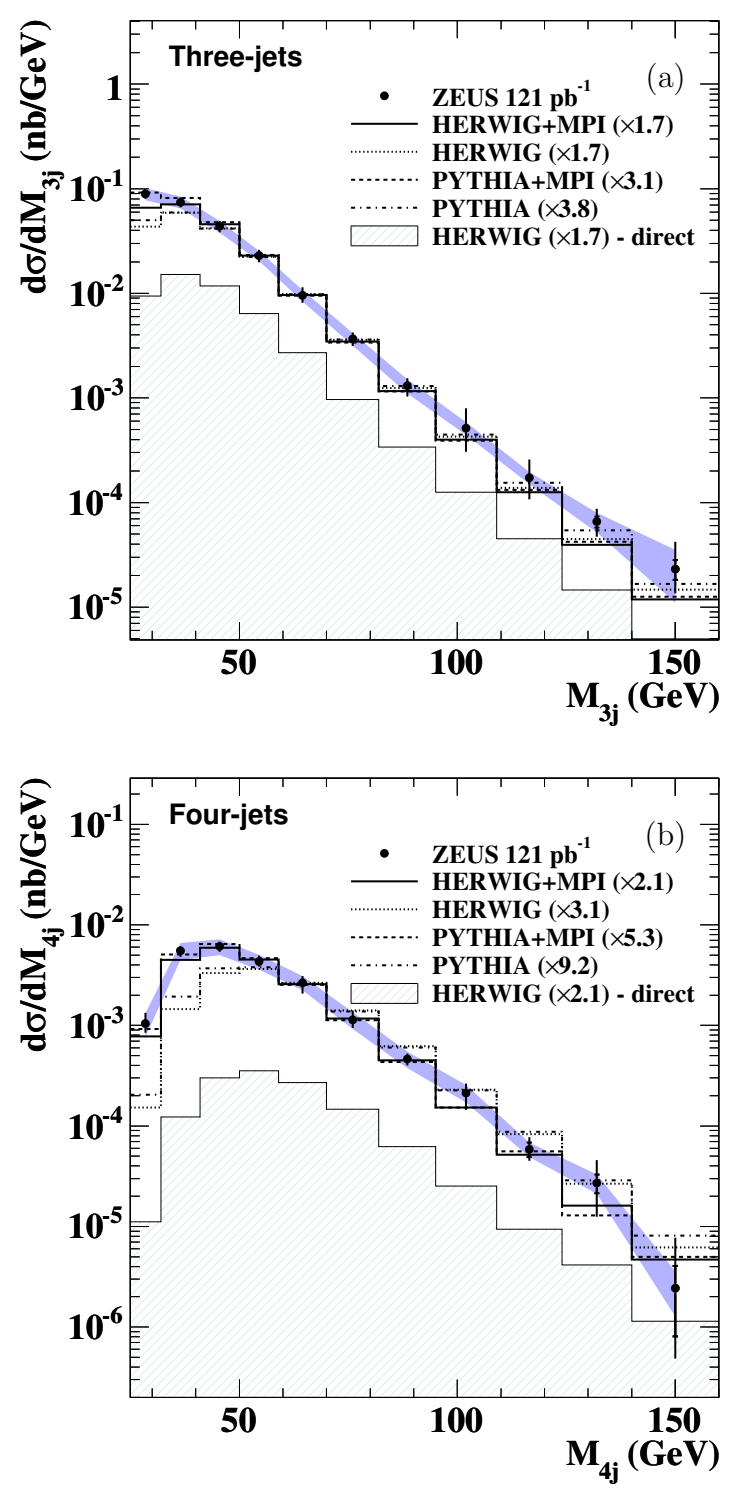

Figure 3. 3- and 4-jet cross-sections as a function of the invariant mass of the jet system. The QCD models and the errors of the data points are described in the text. [6] only described by the models, if MI are included in the simulation. In that case PYTHIA performs slightly better compared to HERWIG. This behaviour is most significant for the 4-jet final state, where the contribution from resolved photon interactions is seen to be much larger, and thus also the probability for remnant-remnant interactions.

The data cross-sections as a function of $x_{\gamma}^{\text {obs }}$ are shown in Fig. 4 for the 3- and the 4-jet final state and the low and high invariant mass regions. Due to the area normalisation of the MC only a shape comparison is possible for the high mass region, and as seen the MI flattens out the distribution slightly. For the low $M_{n j}$ region the data are only described if $\mathrm{MI}$ are included in the MC. It is worth noting that this is most relevant at low $x_{\gamma}^{\text {obs }}$, where the photon's substructure is probed, and remnant-remnant interactions are expected to be most prominent. At $x_{\gamma}^{\text {obs }}$ close to one MI have a comparably small effect on the MC predictions. Again, the effect is largest for the four-jet final state, which is enriched in higher-order reactions.

The presented analysis shows that in order to describe the data MI have to be included in the models. This is most prominent at low invariant masses of the jet system, low $x_{\gamma}^{\text {obs }}$ and for higher jet multiplicity.

\section{Charged Particle Multiplicity in Photo- production}

The H1 collaboration has measured the charged particle multiplicity in photoproduction dijet events. The photoproduction events are selected by tagging the outgoing electron, which puts the upper limit on the photon virtuality, $Q^{2}$, to $0.01 \mathrm{GeV}^{2}$. Analysed are events with at least two jets within the pseudorapidity range $\left|\eta_{\text {jet }}\right|<1.5$ and with transverse momenta $P_{t}^{\text {jet }}>5 \mathrm{GeV}$. Selected are charged particles within the same pseudorapidity range as the jets, $\left|\eta_{\text {part }}\right|<1.5$ and with transverse momenta of $P_{t}>150 \mathrm{MeV}$.

The charged particle multiplicity is measured as a function of the azimuthal difference, $\Delta \phi$, between the leading jet and the particles, as well as a function of $P_{t}^{\text {jet }}$ of the leading jet in different regions of $\phi$. This is done at both low and high 


\section{ZEUS}
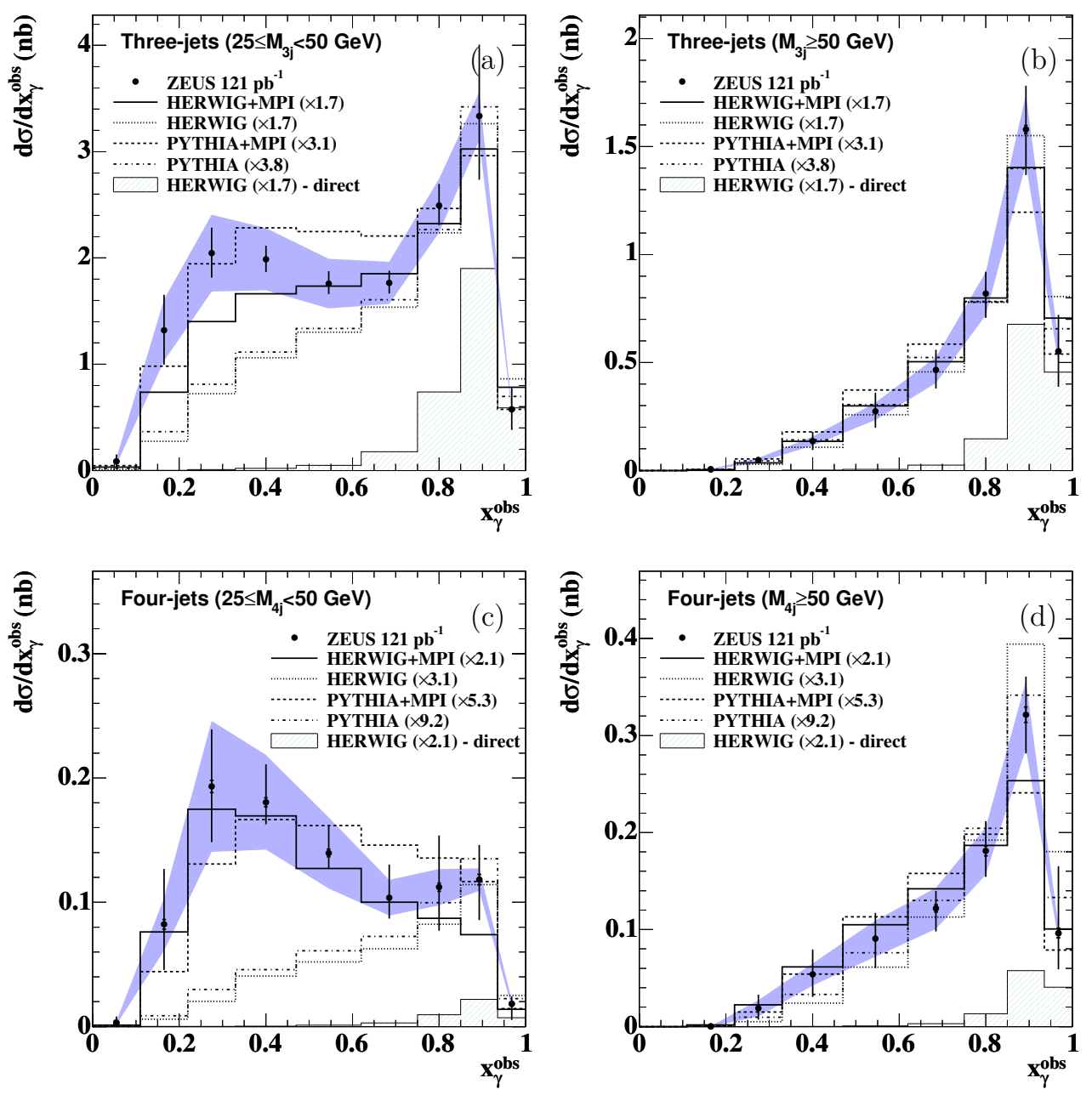

Figure 4. 3- and 4-jet cross-sections as a function of the fractional momentum of the photon carried by the struck quark $\left(x_{\gamma}^{\text {obs }}\right)$. The QCD models and the errors of the data points are described in the text. [6]

$x_{\gamma}^{\text {obs }}$. Four different azimuthal regions are defined (see Fig. 5): The toward region, in which the leading jet axis is always in the center, is defined according to $120^{\circ}<\phi<240^{\circ}$. The away region corresponds to $300^{\circ}<\phi<60^{\circ}$ and, due to momentum conservation, usually contains the subleading jet, although no explicit restriction is made. The transverse regions $240^{\circ}<\phi<300^{\circ}$ and $60^{\circ}<\phi<120^{\circ}$ are distinguished by the scalar sum of the transverse momentum, $P_{t}^{\text {sum }}$, of the charged particles in these angular regions. The high activity region is the region with the high $P_{t}^{\text {sum }}$, while the low activity region has the low $P_{t}^{\text {sum }}$.

In Fig. 6 the charged particle multiplicity is shown as a function of $\Delta \phi$, for a resolved enriched region, $x_{\gamma}^{\text {obs }}<0.7$ (Fig. 6 , upper), and a direct enriched region, $x_{\gamma}^{\text {obs }}>0.7$ (Fig. 6 , lower). These 


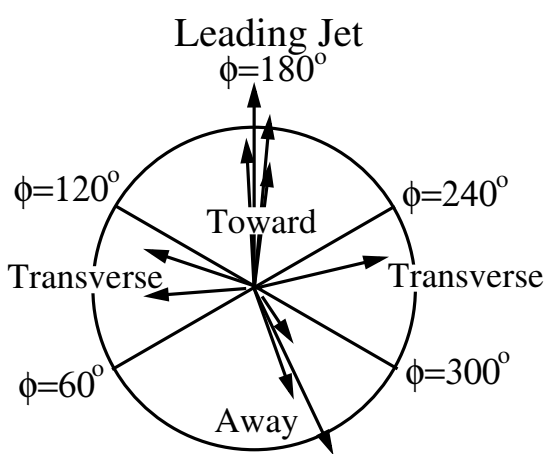

Figure 5. Illustration of the azimuthal regions used in the measurement of the charged particle production in photoproduction [7].

distributions are constructed such that the high activity region is always to the right of the leading jet axis, which is always at $\Delta \phi=180^{\circ}$. Due to momentum conservation the subleading jet is consequently typically to the left in the plot in order to balance the momentum in the high activity transverse region.

The data in Fig. 6 are also compared to MC predictions from PYTHIA 6.2. Both the resolved and direct photon enriched regions are only described, if the average charged particle multiplicity in the MC is increased by including $\mathrm{MI}$ in the simulation. This effect is significantly more prominent in the resolved enriched region. In each region the $\mathrm{MI}$ increase the activity in the $\mathrm{MC}$ by roughly an equal amount of charged particles over the full $\Delta \phi$ range, except in the region of the subleading jet, where the contribution from MI is smaller.

In Figs. 7 and 8 the average charged particle multiplicity is shown as a function of the transverse momentum of the leading jet, $P_{t}^{\text {jet }}$, for the different azimuthal regions. Again the measurements are separated into a direct and a resolved enriched region. Looking at the data one can conclude that in the toward and away regions the charged particle multiplicity increases with $P_{t}^{\text {jet }}$, but the opposite is seen in the transverse regions. Again, the data are only described by the MC if MI are included in the simulation. The contribu-
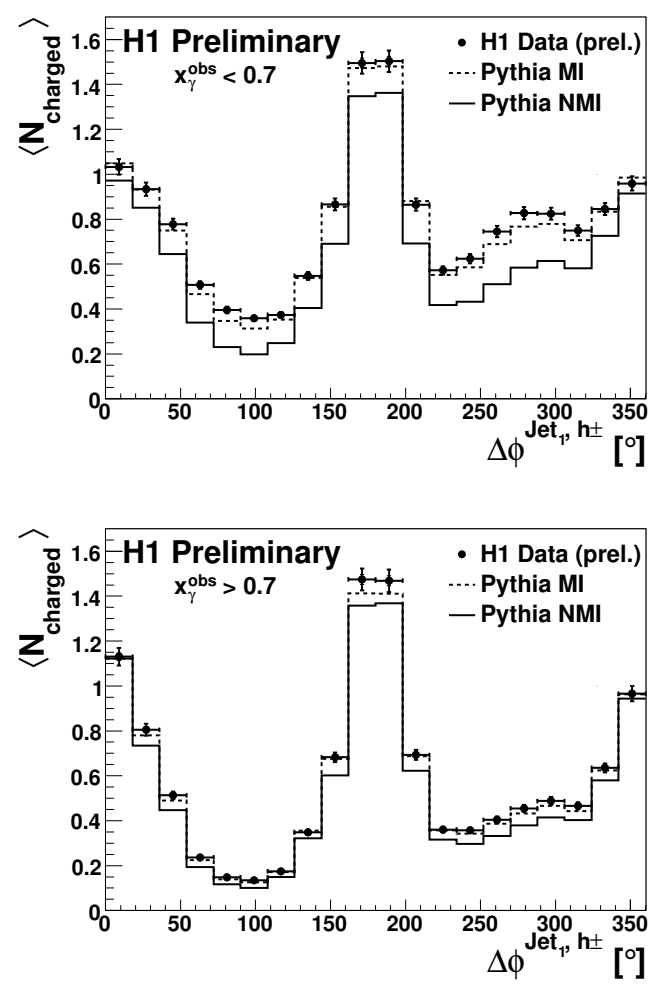

Figure 6. Charged particle multiplicity as a function of the azimuthal difference between the leading jet and the charged particles. The figures are constructed such that the leading jet is centred at $\Delta \phi=180^{\circ}$, and the high activity region is always to the right. The data are compared to PYTHIA with and without multiple interactions [7].

tion from the MI is not constant in the MC but increases slightly with lower $P_{t}^{\text {jet }}$ and lower $x_{\gamma}^{\text {obs }}$.

The description of the data by the MC was improved by tuning of the MI model in PYTHIA. The parameters ${ }^{1}$ which determine the length of the color strings in case of MI were varied. At the TEVATRON such tunes have been performed extensively to different data, e.g. [16]. While the TEVATRON data suggest MI with short color strings, the description of the HERA data pre-

$\overline{{ }^{1} \mathrm{PARP}(85) \text { and } \operatorname{PARP}}(86)$ 

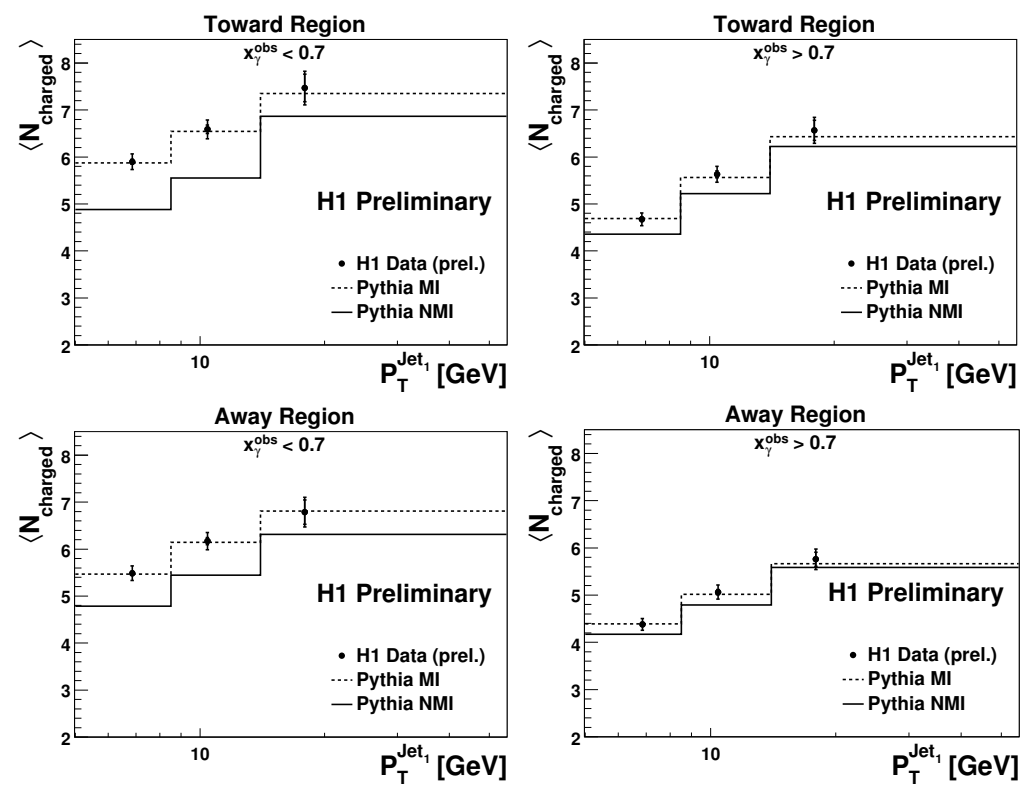

Figure 7. The multiplicity of charged particles in photoproduction in the toward and the away azimuthal regions, as a function of the transverse momentum of the leading jet in the toward region. [7]
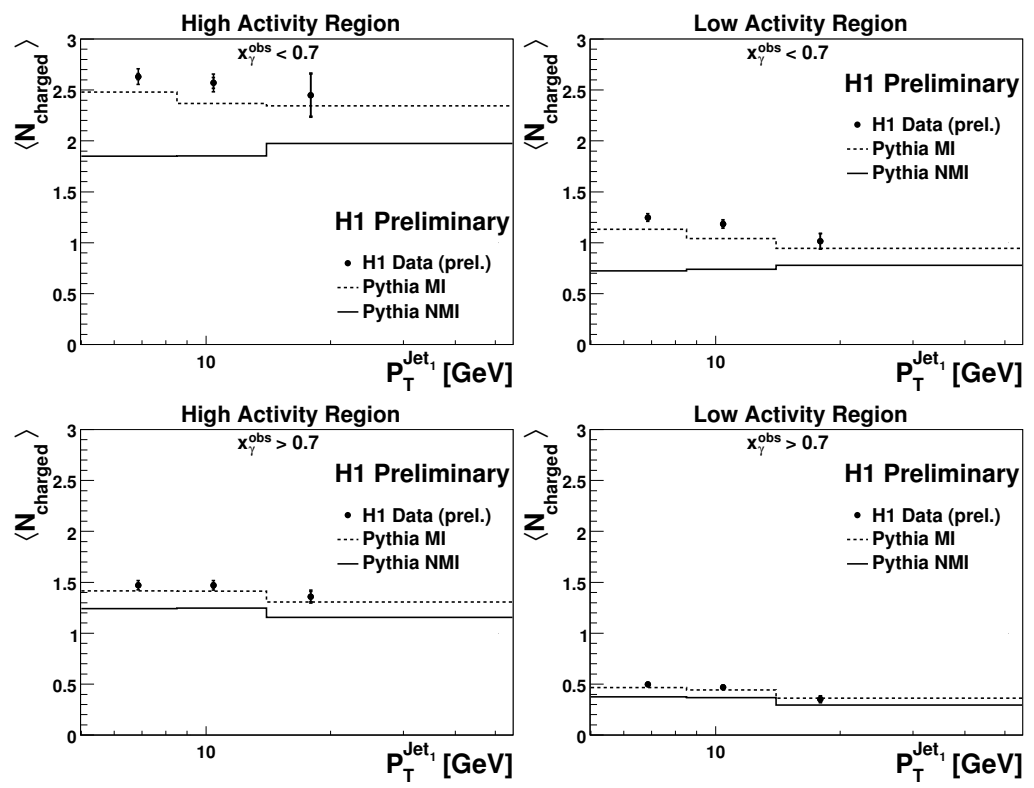

Figure 8. The multiplicity of charged particles in photoproduction in the high and low activity transverse azimuthal regions, as a function of the transverse momentum of the leading jet in the toward region. [7] 
sented here is improved when the long color string scenario is used.

An attempt to describe the average charged particle multiplicity, without including multiple interactions in the MC, was made by increasing the maximum allowed virtuality ${ }^{2}$ of the final state radiation by a factor of 4 (not shown). The result was only a relatively small increase of the charged particle multiplicity in the MC predictions. Furthermore, the description of the shape as a function of $P_{t}^{\text {jet }}$ did not improve significantly.

The presented analysis shows that the charged particle multiplicity in dijet events in photoproduction is only described by the MC, if $\mathrm{MI}$ is included in the MC generated events. This is most prominent at low $x_{\gamma}^{\text {obs }}$ and for low transverse momentum of the leading jet.

\section{Minijet Multiplicities in DIS}

H1 analysed DIS events within the kinematical range $5<Q^{2}<100 \mathrm{GeV}^{2}, 0.1<y<0.7$ and $W>200 \mathrm{GeV}$, where the requirement on the invariant mass of the hadronic final state, $W$, enhances the production of soft jets and events with low $x_{\mathrm{Bj}}$. The results are presented for an inclusive jet sample, containing at least one jet, and a dijet sample in which the subleading jet $(s j)$ is required to be roughly back-to-back to the leading jet $(l j)$ according to $\left|\Delta \phi^{*}\right|=\left|\phi_{l j}^{*}-\phi_{s j}^{*}\right|>140^{\circ}$, where $\phi^{*}$ denotes the azimuthal angle in the hadronic center of mass system. The jets are required to fulfil $E_{T}>5 \mathrm{GeV}$ and $0.5<\eta_{\text {jet }}^{\text {lab }}<2.79$. The $E_{T}$ requirement is applied in both the laboratory and the hadronic center of mass frame.

The average multiplicity of jets with $E_{T}>$ $3 \mathrm{GeV}$, referred to as minijets, is measured in different azimuthal regions relative to the leading jet. These regions are sketched in Fig. 9 and are defined for each event in the same way as described for the photoproduction measurement from H1. Although with the difference that $20^{\circ}$ buffer regions are used between the away region and the transverse regions in order not to contaminate the latter ones with particles belonging to the subleading jet.

\footnotetext{
$\overline{{ }^{2} \mathrm{PARP}(67) \text { was increased from }} 1.0$ to 4.0
}

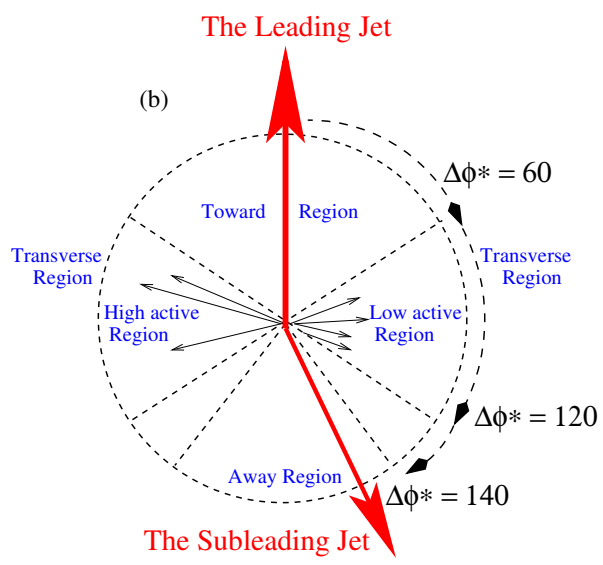

Figure 9. Illustration of the transverse regions used for the measurement of the minijet multiplicity in DIS. [8]

In Fig. 10 the average minijet multiplicity as a function of the transverse momentum of the leading jet is presented for the inclusive jet sample. This is done in bins of $Q^{2}$ and for the different azimuthal regions.

The Fig. 10 (left) shows comparisons to RAPGAP and CDM. The predictions from RAPGAP contain contributions from both direct and resolved photon events. Neither RAPGAP nor CDM have any MI implemented, and in the transverse regions, where MI signals are expected to be important, these models predict too low a minijet multiplicity. This failure is most significant at low $Q^{2}$, where the contribution from resolved photons is large, and thus remnant-remnant interactions are expected to be relevant. In the toward and the away region, where hard physics dominates, the data are reasonably well described.

Fig. 10 (right) shows the same data compared to PYTHIA 6.2, with and without MI. In the low $Q^{2}$ bins, where the resolved component is large, the inclusion of MI events provides a clear improvement of the description of the data in the transverse regions, while at high $Q^{2}$ the MI make only a very small difference. It should be kept in mind that in PYTHIA 6.2, only remnant-remnant interactions are simulated. This means that for 

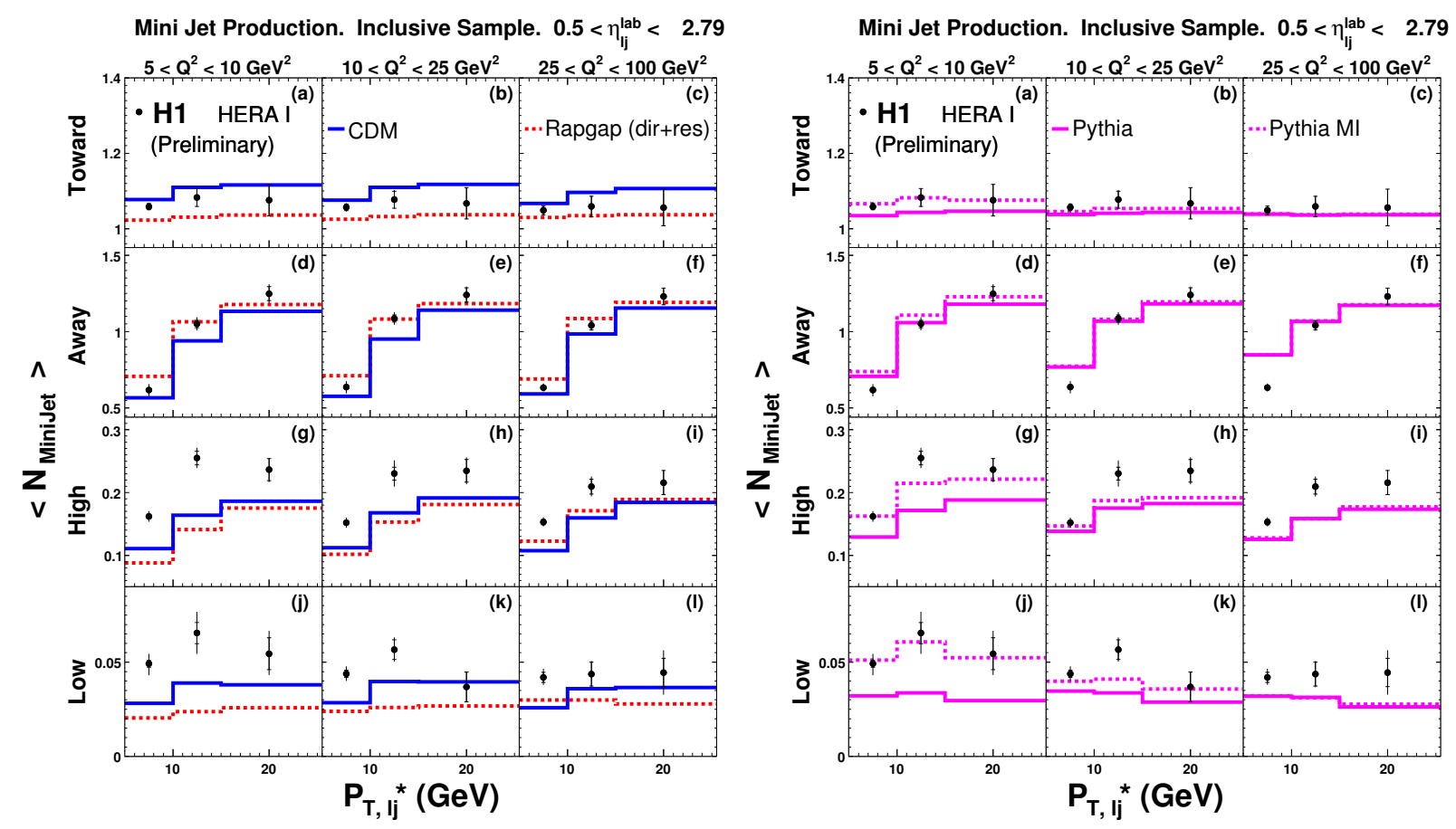

Figure 10. Average minijet multiplicity in bins of $Q^{2}$ in different azimuthal regions with respect to the leading jet, as a function of the transverse momentum of the leading jet in the inclusive jet sample. The data are compared to QCD models which are described in the text. [8]

the direct contribution MI are missing in the simulation. Thus the fact that PYTHIA 6.2 with and without MI give about the same prediction in the highest $Q^{2}$ bin reflects the fact that there is very little contribution from resolved photons in this kinematic region.

\section{Conclusion}

Measurements from the ZEUS and H1 experiments on 3- and 4-jet cross-sections [6], charged particle multiplicity in photoproduction [7] and minijet multiplicity in DIS [8] provide some indication that MI have to be considered in order to describe the data. In the multijet analysis in photoproduction this is most significant for low invariant masses of the jet system, and when the event contains 4 hard jets rather than 3. It is also observed that the fixed-order calculation only describes the photoproduction data after the large corrections for MI have been applied. The charged particle multiplicity analysis in photoproduction shows that the activity in the UE depends on the transverse momentum of the leading jet.

In the analysis of minijet production in DIS the contribution from remnant-remnant $\mathrm{MI}$ is largest at low $Q^{2}$ and shows up in the azimuthal regions transverse to the leading jet. At high $Q^{2}$ where the contribution from the resolved photon is small, and remnant-remnant interactions are thus strongly suppressed, the data description is not improved when the available MI model is included in the MC. This may reflect the fact that a theoretical model for $\mathrm{MI}$ in direct photon interactions is currently not implemented in the $\mathrm{MC}$ generators used.

The analyses presented here comprise several more results. The 3 - and 4 -jet cross-sections in photoproduction have been measured as a func- 
tion of several additional variables. For the minijet production in DIS results exist also for more inclusive rapidity regions as well as a dijet sample.

The overall message is that the activity in the events, in terms of jet multiplicity and the soft underlying event, needs to be increased in the models by adding MI. It is however not clear, if the data presented cannot be described in other ways, e.g. by parton showers based on other evolution equations than DGLAP.

\section{REFERENCES}

1. S. Aid et al. [H1 Collaboration], Z. Phys. C 70 (1996) 17.

2. T. Ahmed et al. [H1 Collaboration], Nucl. Phys. B 445 (1995) 195 [arXiv:hepex/9504004].

3. J. Breitweg et al. [ZEUS Collaboration], Eur. Phys. J. C 1109 (1998) 109 [arXiv:hepex/9710018].

4. T. Sjöstrand, L. Lönnblad and S. Mrenna, [arXiv:hep-ph/0108264].

5. G. Marchesini et al. Comput. Phys. Commun. 67 (1992) 465.

6. S. Chekanov et al. [ZEUS Collaboration], Nucl. Phys. B 792 (2008) 1 [arXiv:0707.3749 [hep-ex]].

7. H1 Collaboration, "Study of Multiple Interactions in Photoproduction at HERA", presented at MPI@LHC08, Perugia, Italy, H1prelim-08-036

8. S. Osman, "Mini-Jets in Deep Inelastic Scattering at HERA", Proc. of 15th Int. Workshop on Deep-Inelastic Scattering and Related Subjects, Munich, April 2007, http://dx.doi.org/10.3360/dis.2007.192.

9. A.A. Affolder et al. [CDF Collaboration], Phys. Rev. D 65 (2002) 092002.

10. H. Jung, Comput. Phys. Commun. 86 (1995) 147.

11. L. Lönnblad, Comput. Phys. Commun. 71 (1992) 15.

12. T. Sjöstrand and P.Z. Skands, JHEP 0403 (2004) 053 [arXiv:hep-ph/0402078].

13. J.M. Buttherworth and M.H. Seymour, "JIMMY4: Multiparton Interactions in HERWIG for the LHC" (unpublished), 2005, http://hepforge. cedar.ac .uk/jimmy/.

14. M. Klasen, T. Kleinwort and G. Kramer, Eur. Phys. J. direct C 1 (1998) 1.

15. M. Klasen, Eur. Phys. J. C 7 (1999) 225.

16. R. Field, "Minimum bias and underlying event at the Tevatron and the LHC", presented at the Fermilab MC workshop, Oct. 4, 2002, http://www.phys.ufl.edu/ ${ }^{\sim r f i e l d / c d f / ~}$ 